\title{
D-SPECT: New technology, old tricks
}

\author{
Yung Hsiang Kao, MBBS, FAMS, ${ }^{a}$ and Nathan Better, MBBS, FRACP, ${ }^{a, b, c}$ \\ a Department of Nuclear Medicine, The Royal Melbourne Hospital, Melbourne, VIC, Australia \\ ${ }^{b}$ Department of Cardiology, The Royal Melbourne Hospital, Melbourne, Australia \\ c University of Melbourne, Melbourne, Australia
}

Received Sep 4, 2015; accepted Sep 4, 2015

doi: 10.1007/s12350-015-0290-5

\section{See related article, pp. 301-310}

The dedicated cardiac cadmium-zinc-telluride (CZT) camera is the latest major technological advancement in Nuclear Cardiology after Tc-99 mbased myocardial perfusion tracers, gated single-photon emission computed tomography (SPECT) imaging, and iterative reconstruction. ${ }^{1}$ The high performance of dedicated cardiac CZT cameras is due to the integration of solid-state semiconductor detectors, cardiac-centric collimator choice, and geometrical setup and improvements in image reconstruction. ${ }^{2}$ Depending on the injected radiotracer activity, dedicated cardiac CZT cameras may achieve 5-10 times higher count sensitivity and 3-6 times faster scanning times than standard sodium iodide (NaI) SPECT cameras. ${ }^{2}$

The main dedicated cardiac CZT cameras commercially available are the Discovery NM series (GE Healthcare) and the Spectrum Dynamics (D-SPECT). The diagnostic performance of the Discovery NM 530c was found to be comparable to that of standard $\mathrm{NaI}$ SPECT with the advantage of superior image quality and shorter acquisition time. ${ }^{3}$ In terms of diagnostic accuracy referenced to coronary angiography, the DSPECT with upright-supine acquisition and quantitative perfusion analysis achieved a sensitivity of $94 \%$ and specificity of $86 \%$ for angiographically significant coronary artery stenosis. ${ }^{4}$ Furthermore, the Discovery NM 530c was found to correctly diagnose more patients

Reprint requests: Nathan Better, Department of Nuclear Medicine, The Royal Melbourne Hospital, Ground Floor, Clinical Sciences Building, Grattan Street, Parkville, Melbourne, VIC, 3050, Australia; nathan.better@mh.org.au

J Nucl Cardiol 2016;23:311-2.

$1071-3581 / \$ 34.00$

Copyright (C) 2015 American Society of Nuclear Cardiology. with multi-vessel disease than standard NaI SPECT, referenced to coronary angiography. ${ }^{5}$

However, with new technology comes new technical traps that may adversely affect diagnostic accuracy for any new user. Therein lies the intangible asset of clinical experience in the usage of any new medical technology. Artifacts of dedicated cardiac CZT cameras are routinely encountered in daily clinical practice, but are scarcely described in the literature. Recognition of common technical artifacts and the different methods available to overcome them is vital to overall diagnostic accuracy. Over time, a new user of a dedicated cardiac CZT camera will slowly gain experience and familiarity with its technical traps, although an apprenticeship in an experienced center may ameliorate the learning curve. These traps will be different not only from the NaI SPECT cameras, but also between the different CZT technologies.

In this issue of the Journal of Nuclear Cardiology, Allie et al describes the common pitfalls and artifacts of the D-SPECT dedicated cardiac CZT camera. ${ }^{6}$ Their paper is based on single-center experience and is largely qualitative, but is nevertheless a useful resource for those new to cardiac CZT technology on the recognition and troubleshooting of common technical artifacts in clinical practice.

In general, technical artifacts in myocardial perfusion scintigraphy may be classified into radiotracer, camera hardware, software, and patient-related factors. Rigorous adherence to protocol is essential to ensure a diagnostic quality test. Each institution must optimize their protocol based on their experience, camera type, clinical workflow, and patient demographics.

Soft tissue attenuation is one of the most common causes of artifacts. The soft tissue involved is usually due to obesity, breast tissue, or the diaphragm. An apparent perfusion defect in a typical location, demonstrating normal wall motion, changing appearance with patient repositioning, which does not conform to an expected coronary arterial territory would favor 
attenuation artifact. Re-scanning after patient repositioning is a simple method to discern true perfusion defects from such attenuation artifacts. This is because the soft tissues that surround the heart may shift slightly with different patient positions leading to different degrees of attenuation between scans. However, this strategy is cumbersome for standard NaI SPECT cameras, as each acquisition requires around 15 minute to complete, slightly less if commercially available software such as resolution recovery is used. In contrast, ultra-fast dedicated cardiac CZT cameras can complete an acquisition in 3-6 minute, making re-scanning in multiple positions feasible even in high-throughput centers. The Discovery NM series has a horizontal table which allows supine and prone imaging. The DSPECT takes this one step further by using an adjustable chair which also allows for semi-reclining prone scanning in addition to supine and semi-upright. ${ }^{7}$ Allie et al provide some examples of breast and diaphragmatic attenuation artifacts which illustrates the benefits of patient repositioning. ${ }^{6}$ Notably, the Discovery NM/CT 570c (GE Healthcare) camera has an integrated $\mathrm{CT}$ which provides an efficient platform for $\mathrm{CT}$ attenuation correction, coronary calcium scoring, or even CT coronary angiography in the same sitting. The added cost of such a system limits its availability, and both the Discovery series and D-SPECT can use CT data acquired on another camera to be integrated with the SPECT imaging from the CZT acquisition.

Patient motion is another common cause of artifacts. The ultra-fast dedicated cardiac CZT camera has diminished, but not eliminated, the likelihood of patient motion during acquisition. On standard NaI SPECT cameras, patient motion may be detected by carefully reviewing the rotating cine display. However, this approach is not feasible for dedicated cardiac CZT cameras due to different hardware setup. Instead, dedicated cardiac CZT cameras rely on sinogram analysis for motion detection, and some examples are provided by Allie et al. ${ }^{6}$ On NaI SPECT cameras, minor motion artifacts may be managed by motion-correction software suited to different camera systems, while significant motion usually requires repeat imaging. On the CZT systems, repeat imaging is required, but, as pointed out, can be quickly performed. Allie et al also have examples of other artifacts, e.g., detector failure, sub-diaphragmatic scatter, and region-of-interest delineation, which will be useful for new users of the D-SPECT. ${ }^{6}$

The cardiac-centric design of dedicated cardiac CZT cameras confers many advantages, but is also its Achilles heel. It may be difficult to position the heart of a very large patient in the center of the field-of-view. This may cause artifact resembling myocardial hypoperfusion which must be recognized at the time of acquisition and processing to avoid misdiagnosis. In contrast, standard NaI SPECT cameras (not using cardiofocal collimation) do not usually have this problem due to externally rotating gamma camera heads which can accommodate very large patients.

Another disadvantage of dedicated cardiac CZT cameras is its lack of versatility for general Nuclear Medicine imaging, and is therefore feasible primarily in centers with a high Nuclear Cardiology throughput. Dedicated cardiac CZT cameras may be beyond the reach of most regional or remote Nuclear Medicine centers, which often have only one gamma camera for all forms of scintigraphy. Even metropolitan Nuclear Medicine centers may not have sufficient Nuclear Cardiology throughput to economically justify the purchase and running costs of a dedicated cardiac CZT camera. We welcome the manuscript of Allie and colleagues ${ }^{6}$ as a valuable reference for the new user of this technology. New technology is always exciting, but let's not forget that the versatility of the standard NaI SPECT camera has made it the workhorse of Nuclear Medicine for decades, and will likely remain so for many more years to come.

\section{Conflict of interest}

The authors declare no conflicts of interest.

\section{References}

1. Miller TD, Askew JW, O'Connor MK. New toys for nuclear cardiologists. Circ Cardiovasc Imaging 2011;4:5-7.

2. Garcia EV, Faber TL, Esteves FP. Cardiac dedicated ultrafast SPECT cameras: New designs and clinical implications. J Nucl Med 2011;52:210-7.

3. Esteves FP, Raggi P, Folks RD, Keidar Z, Askew JW, Rispler S, et al. Novel solid-state-detector dedicated cardiac camera for fast myocardial perfusion imaging: Multicenter comparison with standard dual detector cameras. J Nucl Cardiol 2009;16:927-34.

4. Nakazato R, Tamarappoo BK, Kang X, Wolak A, Kite F, Hayes SW, et al. Quantitative upright-supine high-speed SPECT myocardial perfusion imaging for detection of coronary artery disease: Correlation with invasive coronary angiography. J Nucl Med 2010;51:1724-31.

5. Gimelli A, Bottai M, Giorgetti A, Genovesi D, Kusch A, Ripoli A, et al. Comparison between ultrafast and standard single-photon emission CT in patients with coronary artery disease: A pilot study. Circ Cardiovasc Imaging 2011;4:51-8.

6. Allie R, Hutton BF, Prvulovich E, Bomanji J, Michopoulou S, Ben-Haim S. Pitfalls and artifacts using the D-SPECT dedicated cardiac camera. J Nucl Cardiol 2015. doi:10.1007/s12350-015-0277-2.

7. Perrin M, Djaballah W, Moulin F, Claudin M, Veran N, Imbert L, et al. Stress-first protocol for myocardial perfusion SPECT imaging with semiconductor cameras: High diagnostic performances with significant reduction in patient radiation doses. Eur J Nucl Med Mol Imaging 2015;42:1004-11. 